\title{
Intranasal insulin therapy: the clinical realities
}

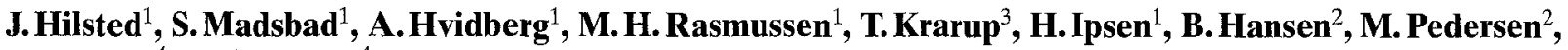 \\ R. Djurup ${ }^{4}$, B. Oxenbøll ${ }^{4}$ \\ ${ }^{1}$ Department of Internal Medicine and Endocrinology, Hvidovre Hospital, University of Copenhagen, Copenhagen, Denmark \\ ${ }_{2}^{2}$ Department of Oto-Rhino-Laryngology, Rigshospitalet, University of Copenhagen, Copenhagen, Denmark \\ ${ }^{3}$ Department of Internal Medicine, Køge Hospital, Copenhagen, Denmark \\ ${ }^{4}$ Diabetes Care Division, Novo-Nordisk A/S, Copenhagen, Denmark
}

Summary To evaluate metabolic control and safety parameters (hypoglycaemia frequency and nasal mucosa physiology), 31 insulin-dependent diabetic patients were treated with intranasal insulin at mealtimes for 1 month and with subcutaneous fast-acting insulin at meals for another month in an open, crossover randomized trial. During both treatment periods the patients were treated with intermediate-acting insulin at bedtime. Six of the patients were withdrawn from the study during intranasal insulin therapy due to metabolic dysregulation. Serum insulin concentrations increased more rapidly and decreased more quickly during intranasal as compared with subcutaneous insulin administration. Metabolic control deteriorated, as assessed by haemoglobin $\mathrm{A}_{1 \mathrm{c}}$ concentrations, slightly but significantly after intrana- sal as compared with subcutaneous insulin therapy. The bioavailability of intranasally applied insulin was low, since intranasal insulin doses were approximately 20 times higher than subcutaneous doses. The frequency of hypoglycaemia was similar during intranasal and subcutaneous insulin therapy, and nasal mucosa physiology was unaffected after intranasal insulin. We conclude that due to low bioavailability and to a high rate of therapeutic failure, intranasal insulin treatment is not a realistic alternative to subcutaneous insulin injections at the present time. [Diabetologia (1995) 38: 680-684]

Key words Intranasal insulin administration, absorption enhancers, metabolic control, subcutaneous insulin administration.
The results of the DCCT (Diabetes Control and Complications Trial) have finally provided proof of the relationship between blood glucose control and the rate of development of long-term diabetic complications. However, in the DCCT, normalization of haemoglobin $\mathrm{A}_{1 \mathrm{c}}$ concentrations was achieved for only $5 \%$ of the patients, despite intensive insulin therapy involving multiple insulin injections [1]. Clearly, there is a need for further improvement of insulin therapy.

Received: 16 June 1994 and in revised form: 28 November 1994

Corresponding author: Dr. J.Hilsted, Department of Internal Medicine and Endocrinology, Hvidovre University Hospital, DK-2650 Hvidovre, Denmark

Abbreviations: IDDM, Insulin-dependent diabetes mellitus; NIDDM, non-insulin-dependent diabetes mellitus.
The limitations of intensive insulin therapy are mainly due to the pharmacokinetic properties of subcutaneously injected insulin, resulting in an increased frequency of hypoglycaemia [2]. Whereas avoiding hypoglycaemia with intensive insulin therapy only seems possible by raising blood glucose levels, a more physiological insulin administration might potentially reduce blood glucose without increasing the frequency of hypoglycaemia [3]. In this context, intranasal insulin administration has gained considerable interest, since after intranasal application of insulin, plasma insulin concentrations increase more rapidly and return to basal levels more quickly than after subcutaneous insulin injections [4]. Furthermore, this "needle-free" way of administering insulin avoids the problems inherent in insulin injections.

Clinical data on intranasal insulin therapy are relatively scarce, since previous investigations were either pharmacokinetic studies, carried out in healthy 
Table 1. Clinical characteristics of the subjects studied

\begin{tabular}{lcc}
\hline & $\begin{array}{l}\text { Patients } \\
\text { completing } \\
\text { the study } \\
(n=19)\end{array}$ & $\begin{array}{l}\text { Patients with- } \\
\text { drawn during } \\
\text { the study }(n=12)\end{array}$ \\
\hline Age (years) & $32 \pm 2$ & $31 \pm 3$ \\
Height $(\mathrm{cm})$ & $177 \pm 2$ & $173 \pm 3$ \\
Body weight $(\mathrm{kg})$ & $73 \pm 3$ & $71 \pm 3$ \\
Body mass index $\left(\mathrm{kg} / \mathrm{m}^{2}\right)$ & $23 \pm 1$ & $24 \pm 1$ \\
$\begin{array}{l}\text { Duration of insulin } \\
\text { treatment (years) }\end{array}$ & $7 \pm 1$ & $10 \pm 2$ \\
\hline
\end{tabular}

Data given as mean $\pm S E M$

subjects [4-6], or therapeutic studies involving small subsets of patients [7-9]. This paper represents data from a randomized, open, cross-over trial comparing intranasal insulin with subcutaneous soluble insulin as mealtime insulin in 31 insulin-dependent diabetic (IDDM) patients.

\section{Subjects and methodls}

Patients. Thirty-one IDDM patients volunteered for the study after giving informed consent. The study was approved by the Ethics Committee of Copenhagen and Frederiksberg (J.nr. V. $200.2206 / 91$ ). The patients fulfilled the following inclusion criteria: age between 18 and 65 years, soluble insulin given three times or more daily, capability of recognizing hypoglycaemia and no signs or symptoms of other diseases. Exclusion criteria were: allergy of any kind, pregnancy (likely or hoping to become pregnant during the study period), significant abnormalities on rhinoscopy, individual single doses of soluble insulin greater than $25 \mathrm{IU}$, ischaemic heart disease and epilepsy. All patients had fasting C-peptide concentrations below $0.2 \mathrm{nmol} / \mathrm{l}$. Clinical data are given in Table 1.

Study protocol. After a 1-week run-in period in which fasting blood glucose was kept between 7 and $10 \mathrm{mmol} / 1$, the patients were treated for 1 month with multiple insulin injections (soluble insulin three times daily before meals and NPH insulin at bedtime) and for 1 month with intranasal insulin three times a day before meals and NPH insulin at bedtime in a randomized cross-over design. A 1-week run-in period was also included after the first 1 -month treatment period. One half of the intranasal insulin was given just before starting a meal, administered with one half in each nostril. The second half of the dose was given $30 \mathrm{~min}$ later, also as two administrations. The intranasal insulin dose was initially chosen as the equivalent dose of soluble insulin ( $20 \%$ bioavailability expected), and the dose was adjusted during the first week of treatment by daily 4-point home monitoring (preprandial and at 23.00 hours), reviewed by telephone. The therapeutic aim was a blood glucose level between 7 and $10 \mathrm{mmol} / \mathrm{l}$. After the first week of each treatment period, the basal NPH insulin dose remained unchanged, whereas the mealtime insulin dose was adjusted as described. Throughout the study, the patients met in the outpatient clinic at Hvidovre Hospital once a week.

Insulin preparations. Intranasal insulin: Novolin Nasal U500 using lecithin as absorption enhancer. The preparation con- tains per ml: Insulin Human $500 \mathrm{IU}$, glycerol $16 \mathrm{mg}$, didecanoyl-1-alpha-phosphatidylcholine $20 \mathrm{mg}$, cholesterol $2 \mathrm{mg}$, fractionated coconut oil $4 \mathrm{mg}$, monobasic sodium phosphate $0.005 \mathrm{mmol} / \mathrm{l}$, water for injection to make $1 \mathrm{ml}$. Soluble insulin: Insulin Actrapid Human, Novo-Nordisk, Copenhagen, Denmark, $100 \mathrm{IU} / \mathrm{ml}$.

NPH insulin: Insulin Insulatard Human, Novo-Nordisk, $100 \mathrm{IU} / \mathrm{ml}$.

\section{Assessments}

Glycaemic control. At the end of each cross-over period the patients were admitted to the metabolic ward at the Department of Endocrinology, Hvidovre Hospital, for 24-h profiles of blood glucose and serum insulin. These were obtained by constant rate withdrawal of blood from a cubital vein by means of a portable suction device (Vingmed, Copenhagen, Denmark).

After 2 weeks in each cross-over period, each of the patients performed one 7-point home monitored blood glucose profile. Blood samples for the profiles were taken by means of capillary tubes from finger blood drops. The capillary tubes were sent by the patients for laboratory analysis (hexokinase method).

Haemoglobin $\mathrm{A}_{1 \mathrm{c}}$ was measured at the start, at the end of the first and at the end of the second cross-over period.

Hypoglycaemic events. The frequency and severity of hypoglycaemic episodes during the two insulin regimens were monitored by means of patient diary and self-monitoring of blood glucose using the following grading: grade $\mathrm{A}$, mild hypoglycaemic symptoms where no assistance was needed; grade $\mathrm{B}$, hypoglycaemia with more severe symptoms requiring the assistance of another person; grade C, severe hypoglycaemia requiring medical intervention.

Local irritation, mucociliary function and acoustic rhinometry. Before and within 1 week after each treatment period the following parameters were assessed:

Local irritation to the nose after intranasal insulin administration was scored as follows: no irritation $=$ score 0 , slight but acceptable irritation $=$ score 1 , moderate but acceptable irritation $=$ score 2 , unacceptable irritation $=$ score 3 .

Mucociliary function was examined both in vivo and in vitro. The in vivo test consisted of the saccharin particle test, calculating the speed of nasal clearance as the time from application of a saccharin particle to the recognition of a sweet taste divided by the length of the nasal cavity [10]. The in vitro examination was performed by a nasal mucosa biopsy and subsequent measurement of number of 1) ciliated epithelial cells and fraction of cells with immobile cilia and 2) calculation of mean frequency of cilia beats and degree of synchronicity by use of a photometry technique [11].

Acoustic rhinometry was performed to estimate the crosssectional area of the nasal cavity as a function of the distance from the nostril [12]. The total bilateral volume was calculated from the anterior margin of the nostril and $47.7 \mathrm{~mm}$ into the nose. All measurements were done three times. Decongestion was performed with ephedrine $0.25 \%$ spray, two puffs in each nostril repeated after $5 \mathrm{~min}$, and measurement after an additional $10 \mathrm{~min}$.

Assay procedures. Blood glucose and haemoglobin $\mathrm{A}_{1 \mathrm{c}}$ were measured by standard techniques. Serum insulin was measured by the enzyme-linked immunosorbant technique [13]. 
Table 2. Daily doses of nasal/subcutaneous Actrapid and Insulatard

\begin{tabular}{|c|c|c|c|c|c|c|c|}
\hline \multicolumn{8}{|c|}{ Nasal/subcutaneous Actrapid doses (I.U.) } \\
\hline \multicolumn{4}{|c|}{ Novolin Nas. + Insulatard treatment } & \multicolumn{4}{|c|}{ Actrapid $\mathrm{HM}+$ Insulatard treatment } \\
\hline $\begin{array}{c}\text { Week } 1 \\
559 \pm 58\end{array}$ & $\begin{array}{l}\text { Week } 2 \\
661 \pm 77\end{array}$ & $\begin{array}{l}\text { Week } 3 \\
663 \pm 92\end{array}$ & $\begin{array}{l}\text { Week } 4 \\
674 \pm 83\end{array}$ & $\begin{array}{l}\text { Week } 1 \\
38 \pm 3\end{array}$ & $\begin{array}{l}\text { Week } 2 \\
40 \pm 3\end{array}$ & $\begin{array}{l}\text { Week } 3 \\
39 \pm 2\end{array}$ & $\begin{array}{l}\text { Week } 4 \\
39 \pm 2\end{array}$ \\
\hline
\end{tabular}

Insulatard doses (I.U.)

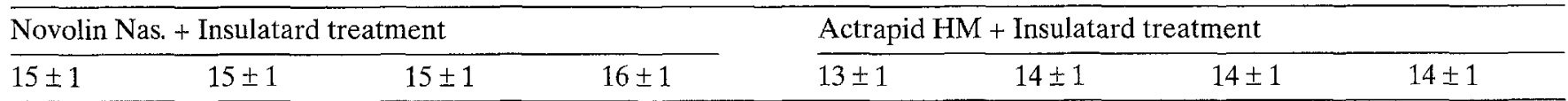

Data are mean \pm SEM

\section{Insulin}

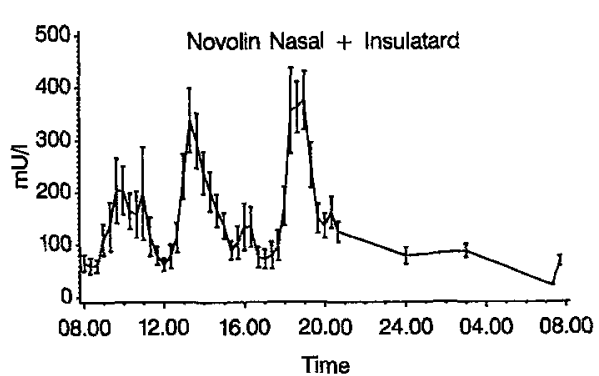

Glucose

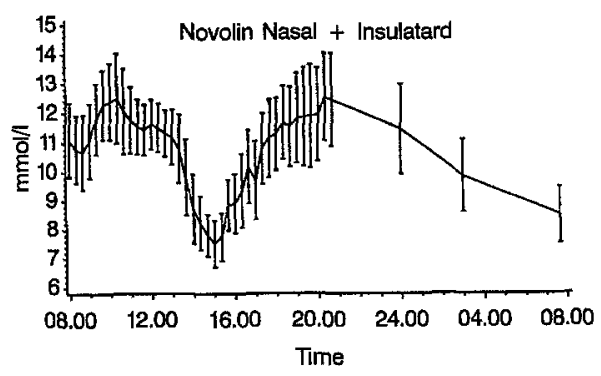

Insulin

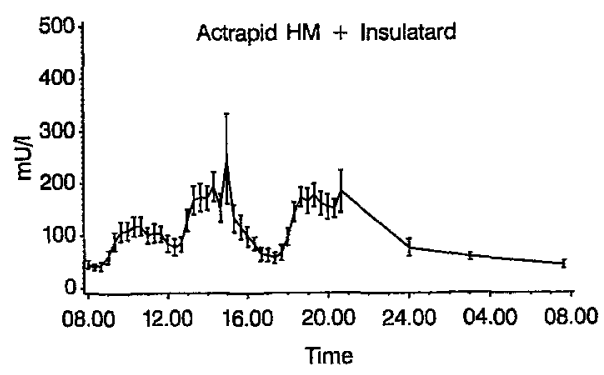

Glucose

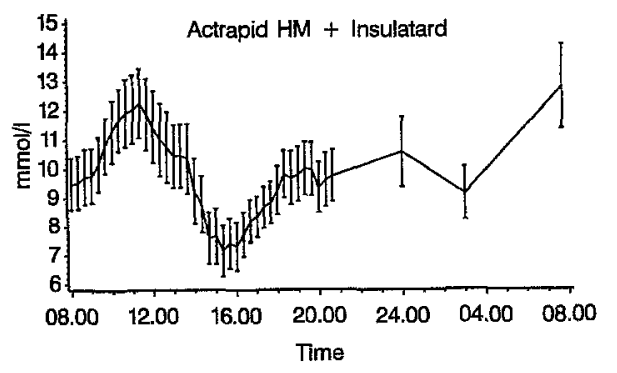

Fig.1. Serum-insulin and blood glucose levels (24-h profiles) during intranasal (left panel) and a subcutaneous (right panel) insulin treatment. Values are mean \pm SEM. For details, see text

\section{Statistical analysis}

Statistical analysis was performed by the Armitage-Hill analysis of incremental areas [14] and by the $t$-test for paired comparisons. Level of statistical significance (two-tailed) was chosen as less than 0.05 .

\section{Results}

Both treatment periods were completed by 19 patients, while 12 patients were withdrawn: 4 patients were withdrawn due to lack of compliance with nasal mucosa investigations, 6 patients were withdrawn due to dysregulation (hyperglycaemia and/or ketonuria), 1 patient experienced severe grade $C$ hypoglycaemia during nasal insulin administration, and 1 patient had to withdraw due to change in occupation. All 6 patients withdrawn due to dysregulation were excluded during the intranasal insulin treatment.
Insulin doses for the patients completing the study are given in Table 2. No significant changes were made in NPH (Insulatard) doses or in soluble insulin (Actrapid) doses throughout the investigation. In order to achieve metabolic control during the run-in period as described (fasting blood glucose 7$10 \mathrm{mmol} / \mathrm{l})$ very large doses of intranasal insulin were required (Table 2 ). These doses did not change significantly during the study period.

Figure 1 shows the 24 -h serum insulin profiles. Serum insulin concentrations rose more rapidly and subsequently returned more quickly to basal levels during intranasal insulin treatment than with subcutaneous insulin injections. Mean blood glucose concentrations during the 24 -h profile period did not differ significantly in the two treatment periods (Fig. 1). In contrast, 7-point home monitoring blood glucose concentrations tended to be higher during intranasal insulin treatment than during subcutaneous insulin administration (Fig. 2). In accordance with the latter observation, haemoglobin $A_{1 c}$ concentrations were 


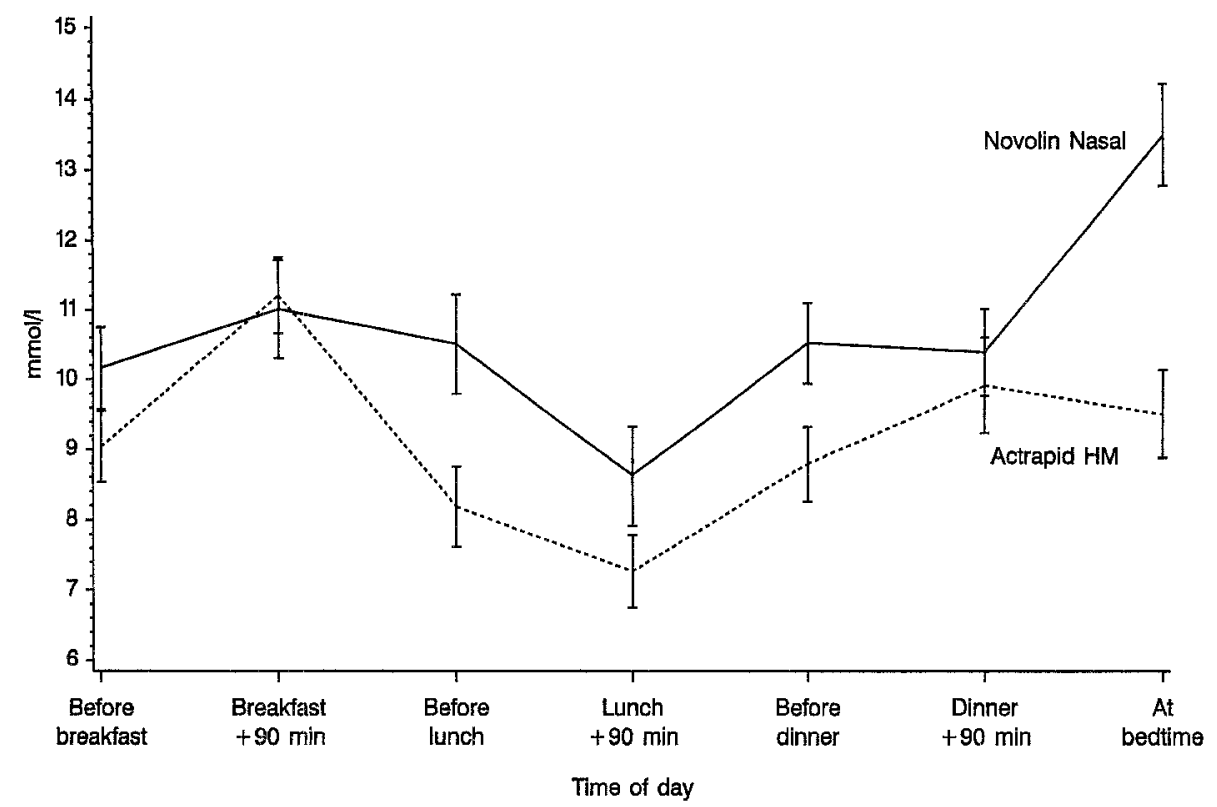

Fig. 2. Seven-point blood glucose home monitored profile during intranasal (solid line) insulin therapy and during subcutaneous (dotted line) insulin therapy. Values are mean \pm SEM. For details, see text slightly but significantly higher after intranasal insulin treatment as compared with after subcutaneous insulin treatment $(8.1$ vs $7.8 \%, P<0.01)$.

The frequency of mild and moderate hypoglycaemia did not differ significantly during the two treatment periods. Only one severe hypoglycaemic period occurred (during intranasal insulin treatment).

No significant changes occurred in mucociliary function or in acoustic rhinometry during the two treatment periods. Acoustic rhinometry expressed as the total bilateral nasal volume showed no significant changes in the two treatment periods before as well as after decongestion of the nasal mucosa. Of 31 patients, 16 had transient nasal irritation after intranasal insulin administration.

\section{Discussion}

It appears from this study that intranasal insulin treatment substituting subcutaneous insulin injections at mealtime may be accomplished in IDDM patients. However, very large doses of intranasal insulin must be applied in order to reach satisfactory metabolic control, suggesting a low bioavailability of the compound, and, in spite of these efforts, metabolic control deteriorated slightly but significantly during intranasal as compared with subcutaneous insulin administration. Thus, intranasal insulin administration is at present not a realistic alternative to subcutaneous insulin injections, at least not using the absorption enhancer and the device employed in the present study.

Intranasal insulin administration results in plasma insulin concentrations which mimic those of healthy subjects to a greater extent than any other hitherto studied route of administration [4]. This was con- firmed in the present study, since plasma insulin concentrations rose more rapidly and returned more quickly to basal levels after intranasal as compared with subcutaneous treatment. This finding in the 24$\mathrm{h}$ profiles was, however, not associated with correspondingly lower blood glucose concentrations, and haemoglobin $\mathrm{A}_{1 \mathrm{c}}$ concentrations did in fact increase slightly during intranasal insulin treatment. This is in accordance with Frauman and co-workers [8] who found a significant increase in haemoglobin $A_{1 c}$ in six patients treated with intranasal insulin for 4 months, as compared with findings in the same subjects after subcutaneous insulin treatment. Notably, in the latter study three of nine patients had to be excluded because a satisfactory metabolic control could not be obtained unless NPH insulin doses at bedtime were increased. Likewise, in our study in which NPH insulin increments were not allowed after the run-in phase 6 of 31 patients were excluded due to dysregulation. It therefore seems that a metabolic control comparable to that of multiple injection regimens is possible in all patients only at the expense of increasing the NPH doses. This dependency on a fairly large basal level of insulin, which seems to be a feature of intranasal insulin treatment, may be the reason why it is seemingly more successful in NIDDM patients $[9,15]$.

Saltzman and co-workers [7] studied eight IDDM patients during 3 months of intranasal therapy as compared with 3 months of subcutaneous insulin treatment. In these patients haemoglobin $\mathrm{A}_{1 \mathrm{c}}$ concentrations did not increase during intranasal insulin treatment, yet these concentrations were fairly high $(8.7 \%)$, supporting our conclusion that near normalization of blood glucose concentrations with intranasal insulin therapy is difficult to obtain, if at all possible. These authors found that nasal irritation was to 
some extent a problem in their patients. In the present study, nasal irritation appeared to be a minor problem, and nasal mucosa physiology did not seem to be affected by intranasal insulin administration for 1 month, since there was no significant change in acoustic rhinometry and mucociliary function in this period.

Due to rapid increments in plasma insulin concentrations, intranasal insulin therapy has been suspected to be associated with hypoglycaemia to a greater extent than subcutaneous insulin treatment. None of the previous clinical investigations nor the present study have confirmed this. Apart from lack of ability to normalize blood glucose concentrations, the main problem associated with intranasal insulin administration in the present study was the low bioavailability. Intranasal insulin doses were approximately 20 times higher than the subcutaneous doses, a figure which has varied from 5 to 20 times in previous investigations, using different absorption enhancers [4-9]. At the present time, solution of the bioavailability problem seems to be crucial for future work in this field.

In conclusion, intranasal insulin administration in association with meals, combined with subcutaneous NPH insulin at bedtime was well tolerated and safe in terms of hypoglycaemic events and nasal mucosa function tests in a 1-month clinical study in 19 IDDM patients, as compared with a 1-month period of subcutaneous insulin treatment at meals. Serum insulin concentration rose more rapidly and returned more quickly to basal levels after intranasal as compared with subcutaneous insulin treatment. However, intranasal insulin doses were about 20 times higher than subcutaneous doses, suggesting a low bioavailability. Despite these large doses, haemoglobin $A_{1 c}$ concentrations rose slightly but significantly during intranasal as compared with subcutaneous insulin treatment. Because of lack of ability to normalize blood glucose concentrations and because of low bioavailability with inherent economic and environmental problems, intranasal insulin therapy is not a realistic alternative to subcutaneous insulin mealtime replacement at the present time.

\section{References}

1. Wang PH (1993) Tight glucose control and diabetic complications. Lancet II: 129

2. Schade DS, Santiago JV, Skyler JS, Rizza RA (1983) Intensive insulin therapy. Medical Examination Publishing, Amsterdam

3. Berger M, Chepper HJ, Chantelau EA, Sonnenberg GE, Joergens V (1982) Absorption kinetics of subcutaneously injected insulin preparations. In: Skyler JS (ed) Insulin update: 1982. Exerpta Medica, Amsterdam, pp 97-110

4. Dreyer K, Vaag A, Bech K, Hansen P, Sørensen AR, Mygind N (1992) Intranasal administration of insulin with phospholipid as absorption inhancer: pharmacokinetics in normal subjects. Diab Med 9: 335-340

5. Pontiroli AE, Alberetto M, Secchi A, Dossi G, Bosi I, Pozza G (1982) Insulin given intranasally induces hypoglycaemia in normal and diabetic subjects. BMJ 284: 303-336

6. Moses AC, Gordon GS, Carey MC, Flier JS (1983) Insulin administered intranasally as an insulin-bile salt aerosol: effectiveness and reproducibility in normal and diabetic subjects. Diabetes 32: 1040-1047

7. Salzman R, Manson JE, Griffing GTet al. (1985) Intranasal aerosolized insulin: mixed-meal studies and long term use in type 1 diabetes. $\mathrm{N}$ Engl J Med 312: 1078-1084

8. Frauman AG, Cooper ME, Parsons BJ, Jerums G, Louis WJ (1987) Long term use of intranasal insulin in insulin-dependent diabetic patients. Diabetes Care 10: 573-578

9. Frauman AG, Jerums G, Louis WJ (1987) Effects of intranasal insulin in non-obese type 2 diabetes. Diabetes Res Clin Pract 3: 197-202

10. Moriarty BG, Robson AM, Smallman LA, Drake-Lee AB (1991) Nasal mucociliary function: comparison of saccharin clearance with ciliary beat frequency. Rhinology 29: 173-179

11. Pedersen M, Sakakura Y, Winther B, Brofeldt S, Mygind N (1983) Nasal mucociliary transport, number of ciliated cells, and beating pattern in naturally acquired common cold. Eur J Respir Dis [Suppl] 128: 355-364

12. Hansen BJ, Osterhammel PA (1991) Morphometrical evaluations of nosecasts compared to acoustic rhinometry. The Journal of Japan Rhinologic Society 30: 124

13. Andersen L, Dinesen B, Jørgensen PN, Poulsen F, Røder ME (1993) Enzyme immunoassay for intact human insulin in serum or plasma. Clin Chem 39: 578-582

14. Armitage P (1971) Statistical methods in medical research. Blackwell, pp 189-217

15. El-Etr M, Slama G, Desplanque N (1987) Preprandial intranasal insulin as adjuvant therapy in type 2 diabetics. Lancet II: $1085-1086$ 